\title{
Video Article \\ Comprehensive Characterization of Extended Defects in Semiconductor Materials by a Scanning Electron Microscope
}

\author{
Ellen Hieckmann ${ }^{1}$, Markus Nacke ${ }^{1}$, Matthias Allardt ${ }^{1}$, Yury Bodrov ${ }^{1}$, Paul Chekhonin ${ }^{2}$, Werner Skrotzki ${ }^{2}$, Jörg Weber ${ }^{1}$ \\ ${ }^{1}$ Institute of Applied Physics, Semiconductor Physics, Technische Universität Dresden \\ ${ }^{2}$ Institut für Strukturphysik, Technische Universität Dresden
}

Correspondence to: Ellen Hieckmann at ellen.hieckmann@tu-dresden.de

URL: https://www.jove.com/video/53872

DOI: doi: $10.3791 / 53872$

Keywords: Engineering, Issue 111, Scanning electron microscope (SEM), silicon, cathodoluminescence (CL), electron beam induced current (EBIC), cross-correlation electron backscatter diffraction (ccEBSD), dislocations, grain boundaries, D-line luminescence

Date Published: 5/28/2016

Citation: Hieckmann, E., Nacke, M., Allardt, M., Bodrov, Y., Chekhonin, P., Skrotzki, W., Weber, J. Comprehensive Characterization of Extended Defects in Semiconductor Materials by a Scanning Electron Microscope. J. Vis. Exp. (111), e53872, doi:10.3791/53872 (2016).

\section{Abstract}

Extended defects such as dislocations and grain boundaries have a strong influence on the performance of microelectronic devices and on other applications of semiconductor materials. However, it is still under debate how the defect structure determines the band structure, and therefore, the recombination behavior of electron-hole pairs responsible for the optical and electrical properties of the extended defects. The present paper is a survey of procedures for the spatially resolved investigation of structural and of physical properties of extended defects in semiconductor materials with a scanning electron microscope (SEM). Representative examples are given for crystalline silicon. The luminescence behavior of extended defects can be investigated by cathodoluminescence $(\mathrm{CL})$ measurements. They are particularly valuable because spectrally and spatially resolved information can be obtained simultaneously. For silicon, with an indirect electronic band structure, CL measurements should be carried out at low temperatures down to $5 \mathrm{~K}$ due to the low fraction of radiative recombination processes in comparison to non-radiative transitions at room temperature. For the study of the electrical properties of extended defects, the electron beam induced current (EBIC) technique can be applied. The EBIC image reflects the local distribution of defects due to the increased charge-carrier recombination in their vicinity. The procedure for EBIC investigations is described for measurements at room temperature and at low temperatures. Internal strain fields arising from extended defects can be determined quantitatively by cross-correlation electron backscatter diffraction (ccEBSD). This method is challenging because of the necessary preparation of the sample surface and because of the quality of the diffraction patterns which are recorded during the mapping of the sample. The spatial resolution of the three experimental techniques is compared.

\section{Video Link}

The video component of this article can be found at https://www.jove.com/video/53872/

\section{Introduction}

It has been known for decades that extended defects exert an influence on the electronic structure of semiconductor materials ${ }^{1-3}$. The effect of extended defects on the performance of electronic devices and other applications such as sensors and solar-cell materials is under extensive experimental and theoretical investigation. Nevertheless, there is no generally accepted theory for the calculation of the electronic states of semiconductors in the presence of extended defects. This is due to the complexity of electronic structure calculations in the case of deviations from the ideal crystal lattice and also to the large diversity regarding the types and configuration of extended defects, as well as the possible combinations among them and with 0 -dim intrinsic and extrinsic defects.

The main types of extended defects are dislocations (1-dimensional defects) and grain boundaries (2-dimensional defects). In the following, we concentrate on both of these types of extended defects in terms of the experiments that can be performed in the scanning electron microscope (SEM). The experimental methods presented here give information about structural, optical and electrical properties of extended defects and, therefore, indirect knowledge of the electronic states in semiconductor materials containing extended defects. The control of the defect-related electronic states is a central issue for the application of semiconductors and the operation of semiconductor devices.

For the structural investigation of extended defects, the electron backscatter diffraction (EBSD) technique can be applied. Usually, an EBSD measurement is performed by point mapping with a stationary electron beam at each point. EBSD then yields information about the crystallographic orientation of the crystal lattice of the sample in the case of single-crystalline material and of the grains in polycrystalline materials. For that purpose the experimentally determined diffraction patterns formed by the Kikuchi bands have to be analyzed by comparison with simulated patterns determined from the crystal space group of the material. If the software for the evaluation of the orientation data is able to calculate the misorientation angle between the crystallographic coordinate systems of neighboring mapping points, the type of grain boundary between them can be determined. If the misorientation angle is smaller than $15^{\circ}$, a low angle grain boundary (LAGB) is present; otherwise it is a high angle grain boundary (HAGB). The type of HAGB is characterized by its $\Sigma$ value where $\Sigma^{-1}$ is the fraction of lattice points lying on a coincidence lattice. So, $\Sigma=3$ stands for the highly symmetric twin boundary ${ }^{4}$. If the EBSD mapping on two planes of the sample surface can be 
measured with an accurate knowledge of the positions of the mappings, the type of the grain boundary plane with Miller indices $h k l$ can also be evaluated by a method proposed by Randle ${ }^{5}$.

Recently, a new procedure for the evaluation of the electron diffraction pattern was derived by Wilkinson et al. ${ }^{6}$ which allows the calculation of all components of the complete local strain tensor, i.e., absolute values of the three normal strain and the three shear strain components. This calculation is performed for each measuring point in a mapping from the corresponding diffraction pattern with respect to a reference pattern taken on an unstrained crystal region with the same crystallographic orientation. This evaluation procedure is based on the determination of small shifts of characteristic features of the EBSD pattern using the cross-correlation technique which gives the name ccEBSD. Relative to a chosen reference point, the strain components and lattice rotations can be measured with precisions of $10^{-4}$ and $0.006^{\circ}$, respectively ${ }^{7}$. Applying ccEBSD measurements in line scans across grain boundaries, or along arrangements of dislocations, one can determine locally the amount as well as the range of the strain fields of these extended defects.

The optical properties of dislocations and grain boundaries can be investigated by spectral and imaging cathodoluminescence (CL) techniques. The luminescence signal is caused by the radiative recombination of electron-hole pairs which are generated in the semiconductor material by the primary electron beam of the SEM. The intensity of the luminescence is proportional to the radiative recombination efficiency which is the ratio of the total minority carrier life time to the radiative recombination time. When this ratio is influenced locally by defects, a contrast in the luminescence distribution can be observed in the CL images. Normally, extended defects act as non-radiative recombination centers and, therefore, the luminescence from band-band-recombination is decreased in the vicinity of extended defects in comparison to the undisturbed semiconductor. However, in the case of $\mathrm{Si}, \mathrm{Ge}$ and some compound semiconductor materials, at dislocations as well as on grain boundaries, characteristic luminescence bands are observed showing photon energies lower than that of the (direct or non-direct) band-to-band recombination in the bulk material ${ }^{8-10}$. As an example, extensive $C L$ investigations of bonded silicon wafers and of multi-crystalline silicon by Sekiguchi and co-workers ${ }^{11-13}$ revealed that dislocations and LAGBs are responsible for the occurrence of shallow and deep levels in the band gap. The corresponding radiative transitions are denoted as $D$ lines in the CL spectra. Nevertheless, the role of the strain field accompanying arrangements of dislocations and of dislocation contamination by oxygen precipitation and transition metal impurities is still controversial for the interpretation of the $\mathrm{D}$ line luminescence. But, if an assignment of the energy position of the luminescence line to a distinct extended defect can be successfully made, then the occurrence of this specific line in the luminescence spectrum can be taken as a signal for the presence of this defect. To increase the luminescence intensity, i.e., the radiative recombination in relation to the non-radiative one, $\mathrm{CL}$ investigations have to be performed at low temperatures (cryo-CL) for semiconductor materials with indirect band structures.

The electrical properties of the extended defects considered here are characterized by imaging the electron beam induced current (EBIC) in the SEM. This current can be observed when electron-hole pairs generated by the primary electron beam are separated by a built-in electric field. This field can be generated by the electric potential of the extended defects themselves or by Schottky contacts on the sample surface. The EBIC image contrast results from local variations of the charge-collection efficiency due to a varying recombination behavior at electrically active defects. The extended defects usually show an increased carrier recombination so that they appear darker in an EBIC image than defect free regions. In the framework of physically based models of defects ${ }^{14}$, a quantitative evaluation of the spatial dependence of the EBIC signal, which is called contrast profile, enables the determination of the minority carrier diffusion length and lifetime as well as the surface recombination velocity. Because these parameters are dependent on temperature, EBIC investigations should also be performed at low temperature (cryo$\mathrm{EBIC}$ ) to obtain an enhanced signal to noise ratio. Alternatively, temperature dependent EBIC measurements enable the determination of the concentration of deep level impurities at dislocations according to a model which was proposed by Kittler and co-workers ${ }^{15,16}$.

It should be noted that the optical and electrical properties of extended defects in semiconductors can be influenced significantly by contamination and by 0 -dim intrinsic defects ${ }^{17}$ which cannot be resolved by scanning electron microscopy. However, the combination of the experimental methods, ccEBSD, CL and EBIC, offers the chance to visualize the extended defects and to quantify their fundamental properties in the SEM. For future applications, where not only failure analysis, but also defect control and defect engineering are intended, this powerful tool will play an important role in the improvement of the performance of semiconductor devices.

\section{Protocol}

\section{Sample Preparation for Cryo-CL Experiment}

1. Use a bulk sample from semiconductor material (here: silicon) with a planar surface, a maximum surface area of $5 \times 5 \mathrm{~mm}$ and thickness in the range between of 0.2 and $0.5 \mathrm{~mm}$.

2. Clean the sample and the sample holder with organic solvents such as ethanol or acetone. Remove the silicon oxide surface layer on the sample by a dip in a solution of hydrofluoric acid (40\%) and de-ionized water in a ratio of 1:10 for $1 \mathrm{~min}$. Flush the sample with de-ionized water.

3. Mill a piece of indium wire mechanically to form an indium foil with a surface area corresponding to that of the sample and with a thickness of about $0.5 \mathrm{~mm}$.

4. Mount the $60^{\circ}$-pre-tilted sample holder on a metallic socket, put the indium foil on the sample holder and the sample on top.

5. Place the socket onto a heating plate.

6. Switch on the heating plate, control temperature of the socket with a thermometer and heat the socket up to $150{ }^{\circ} \mathrm{C}$ so that the melting process of the indium foil is imminent.

Note: It is not necessary that the indium reaches the melting point at $157^{\circ} \mathrm{C}$ but the foil should be ductile.

7. Fix the sample on the indium foil by pressing on the sample with a wooden toothpick for $1 \mathrm{sec}$.

8. Switch off the heating plate and cool down the whole system for about $30 \mathrm{~min}$. 


\section{Sample Preparation for Cryo-EBIC Experiment}

1. Use a bulk sample from semiconductor material (here: silicon) with a planar surface, a maximum surface area of $5 \times 5 \mathrm{~mm}$ and thickness in the range between 0.2 and $0.5 \mathrm{~mm}$.

2. Clean the sample and the sample holder with organic solvents such as ethanol or acetone. Remove the silicon oxide surface layer on the sample by a dip in a solution of hydrofluoric acid (40\%) and de-ionized water in a ratio of 1:10 for 1 min. Flush the sample with de-ionized water.

3. Mount the sample into a metallizing system and evaporate metal (e.g., Al on p-type Si, Au on n-type Si) onto the upper surface of the sample to form a Schottky-contact.

4. Deposit a droplet of fluid gallium-indium eutectic on the backside of the silicon sample and smear it over to produce an ohmic contact.

\section{Sample Preparation for ccEBSD Experiments}

1. Prepare a flat sample surface by applying a sequence of grinding and polishing steps with decreasing particle size of grinding and polishing agents (e.g., first diamond paste $3 \mu \mathrm{m}$ on a sheet of paper, then diamond paste $1 \mu \mathrm{m}$, last polishing on a vibratory polisher with polishing suspension). Flush the sample with de-ionized water between each step.

2. Finish the surface preparation by cleaning the sample in ethanol in an ultrasonic bath for 3 min.

3. Fasten the sample to a metallic sample holder by mechanical fixation or by bonding on a two-sided adhesive carbon tab.

\section{Performing Cryo-CL Experiment}

1. Ensure the availability of cryogens as liquid nitrogen (LN) and liquid helium (LHe) for an experiment duration of $8 \mathrm{hr}$.

2. Use the cryo-stage in the SEM.

3. Insert the light-collecting elliptical mirror from parking position to measuring position into the SEM.

4. Mount a test sample (here: bulk GaAs sample) with a direct band gap transition on the stage.

5. Evacuate the SEM chamber until the column chamber valve opens. Set the SEM imaging parameters acceleration high voltage (HV) to 20 $\mathrm{kV}$, the aperture size to $240 \mu \mathrm{m}$, the high current mode ON, the scan speed of the electron beam to 1 , the magnification to 200 and the noise reduction to pixel average.

6. Use the Everhart Thornley detector for imaging with secondary electrons. Move the stage towards the pole piece until the electron beam can be focused on the sample surface at a working distance (WD) of $15 \mathrm{~mm}$.

7. Run the start-up procedure of the $\mathrm{CL}$ detection system by switching on the power supply for the monochromator, the high-voltage power supply for photomultiplier tube (PMT), the PMT cooling system and the laptop with the CL control program. Start the CL control program and choose measurement of PMT signal vs. time.

8. Set appropriate values for the contrast (maximum) and brightness $(46 \%)$ of the PMT.

9. Create a log file for recording the experiments.

10. Adjust the light-collecting mirror to maximize the integral $C L$ intensity on the test sample by tilting and rotating the mirror. Record a CL test spectrum using the $\mathrm{CL}$ control program.

11. Vent the SEM chamber, remove the test sample, mount the actual sample on indium foil on the sample holder and evacuate the SEM chamber.

12. Link the outlet for cryogenic gases of the SEM with the inlet of a diaphragm pump by flexible vacuum tubes. Use centering rings with an oring as a sealing element and fix it with the clamping rings. Stabilize the system mechanically by integration of a damping weight in the hose coupling.

13. Connect the outlet of the diaphragm pump by a flexible vacuum tube to a He recirculation system if it is available. Use centering rings with an o-ring as a sealing element and fix it with clamping rings.

14. Connect the temperature sensor of the temperature control unit with the appropriate plug connector on the SEM stage. Switch on the power supply of the temperature control unit.

15. Assemble heating tape on the flexible vacuum tube next to the outlet for cryogenic gases of the SEM.

16. Wear protective glasses and protective gloves. Insert the tubing for the liquid He into the LHe Dewar. Connect the outlet of the He transfer tube with the inlet for cryogenic gases of the cryo-stage of the SEM.

17. Set the electron beam parameters HV to $20 \mathrm{kV}$, the aperture size to $240 \mu \mathrm{m}$, the high current mode ON, the scan speed of the electron beam to 1 , the magnification to 200 and the noise reduction to pixel average.

18. Use the Everhart Thornley detector for imaging with secondary electrons. Move the stage towards the pole piece and focus the electron beam onto the sample surface at $\mathrm{WD}=15 \mathrm{~mm}$ for the $\mathrm{CL}$ experiments.

19. Choose the area of interest on the sample surface and scan on the whole region during the entire cooling-down procedure. Ensure that during the entire $C L$ experiment the surface of the sample in the region of interest is at WD = $15 \mathrm{~mm}$ by an appropriate positioning of the stage.

20. Start the cooling-down procedure by entering the lowest target temperature (e.g., $5 \mathrm{~K}$ ) and appropriate parameters for the PID control into the temperature controller according to the technical manual. Open the valve of the LHe transfer tube.

21. Monitor the sample temperature, the He pressure from the diaphragm pump, and the integral CL signal output from the PMT during coolingdown procedure. Reduce the slit width of the monochromator if the CL intensity becomes too high at low temperatures to avoid damaging the PMT.

22. Increase the orifice of the LHe transfer tube if necessary to reach the target temperature.

23. Re-establish the WD $=15 \mathrm{~mm}$ for focused images after reaching the target temperature. Rectify the adjustment of the light-collecting mirror for maximum integral $C L$ intensity on the actual sample.

24. Set appropriate values for the grating (300 lines per $\mathrm{mm}$, blaze at 1,000 nm), spectral region (1,050 to 1,600 nm), step width (5 nm), time per measuring point $(5 \mathrm{sec})$ and slit width $(1 \mathrm{~mm})$ for spectral $\mathrm{CL}$ measurements on the silicon sample. Record CL spectra of the sample using the $\mathrm{CL}$ control program and save them to the hard disk of the laptop. 
25. Choose the planar mirror in the monochromator for panchromatic CL imaging and a blaze grating at particular wavelength for monochromatic $\mathrm{CL}$ imaging, respectively. Adjust the brightness and contrast values of the $\mathrm{CL}$ image in the linear range of the dependence of the grey values from the PMT signal.

26. Adjust appropriate image resolution (e.g., 1,024 x 768 pixels), scan speed and noise reduction method for CL imaging (e.g., for a magnification in the range between 200 and 1,000 the lowest scan speed 14 combined with pixel average, or higher scan speed 8 combined with line average over 20 lines). Record CL images with the SEM control program and save them to the hard disk of the computer.

27. Enter name and parameters of each spectrum and image measured during the whole measuring session into the log file.

28. Switch off the HV of the electron beam and close the SEM column chamber valve before changing the sample temperature by entering a higher target temperature into the temperature controller for temperature dependent CL investigations. Note: This is to avoid a shut-down of the electron gun by instabilities of the SEM chamber vacuum during heating-up.

29. Open the SEM column chamber valve and switch on the HV when the new target temperature is reached and the system vacuum in the SEM chamber is stable again.

30. Switch off the HV of the electron beam, close the SEM column chamber valve, close the shutter for the PMT, switch off the power supply and cooler of the PMT and run the warm-up procedure after finishing the planned CL measurements at low temperatures.

31. Remove the additional devices and tools which have been assembled for the cryo-CL experiment without opening the SEM chamber.

32. Leave the SEM chamber closed for at least $12 \mathrm{hr}$. Check to ensure that the temperature of the sample holder is at RT. Otherwise, heat up the sample holder to RT before opening the SEM chamber.

Note: This is to prevent moisture in the SEM chamber.

33. Remove the sample from the stage and evacuate the SEM chamber.

\section{Performing Cryo-EBIC Experiment}

1. Ensure the availability of LHe for an experiment duration of $8 \mathrm{hr}$.

2. Use the cryo-stage in the SEM.

3. Ensure that a plate of mica is available with an evaporated Au layer on the top surface.

4. Stack on the sample holder first the piece of mica with Au layer upward and then the actual sample on the mica with Schottky-contact upward. Put one contact tip on the Schottky-contact of the actual sample and the other one on the evaporated Au layer of the mica.

5. Evacuate the SEM chamber until the column chamber valve opens. Set the appropriate values of the SEM imaging parameters HV, aperture size, scan speed, magnification and noise reduction.

6. Switch on the sample current amplifier and choose the measurement range so that three decimal places of the specimen current are displayed.

7. Create a log file for recording the experiments

8. Perform the steps 4.12 to 4.16 from the cryo-CL experiments.

9. Set the appropriate values of the SEM parameters HV, aperture size, magnification, scan speed, and noise reduction method for the EBIC imaging (recommended range of parameters for EBIC imaging of grain boundaries are 10 to $20 \mathrm{kV}$ for HV, 20 to $60 \mu \mathrm{m}$ for aperture size, 30 to 1,000 for the magnification, 9 to 14 for scan speed, and pixel or frame averaging).

10. Use the sample current detector for EBIC imaging. Move the stage towards the pole piece and focus the electron beam for a WD in the range between 15 and $25 \mathrm{~mm}$.

11. Choose the area of interest on the sample surface and scan on the whole region of interest during the entire cooling-down procedure.

12. Start the cooling-down procedure by entering the lowest target temperature (e.g., $200 \mathrm{~K})$ and appropriate parameters for the PID control into the temperature controller according to the technical manual. Open the valve of the LHe transfer tube.

13. Monitor the sample temperature and the He pressure from the diaphragm pump during cooling-down.

14. Split the SEM screen and monitor the signal of secondary electrons from the Everart Thornley detector in parallel to the EBIC signal from the sample current detector to watch the variation of the images with temperature.

15. Re-adjust the SEM parameters HV, aperture size, magnification, scan speed, and noise reduction to get EBIC images of high contrast. Readjust also the measuring range of the specimen current amplifier to avoid display overflow at low temperatures.

16. Re-focus the electron beam after reaching the target temperature.

17. Record the EBIC images. Enter name and parameters of each image into the log file.

18. Switch off the HV of the electron beam and close the SEM column chamber valve before changing the sample temperature by entering a higher value of target temperature into the temperature controller for temperature dependent EBIC investigations. Open the SEM column chamber valve and switch on the HV when the new target temperature is reached and the system vacuum is stable again.

19. Switch off the HV of the electron beam, close the SEM column chamber valve and run the warm-up procedure after finishing the planned EBIC measurements at low temperatures.

20. Remove the additional devices and tools which have been assembled for the cryo-EBIC experiment without opening the SEM chamber.

21. Leave the SEM chamber closed for at least $12 \mathrm{hr}$. Check to insure the temperature of the sample holder is at RT. Otherwise, heat up the sample holder to RT before opening the SEM chamber.

22. Remove the sample from the stage and evacuate the SEM chamber.

\section{Performing ccEBSD Experiments}

1. Mount the sample within a small vice or on the pin of a sample holder that is pre-tilted in the range between $65^{\circ}$ and $70^{\circ}$ relative to the electron beam direction.

1. Alternatively, place the sample on the 6 axis eucentric stage in the SEM and tilt the whole stage so that the sample surface normal and the electron beam direction are at an angle in the range between $65^{\circ}$ and $70^{\circ}$. Ensure that the stage will not collide with the detectors and the chamber walls.

2. Evacuate the SEM chamber until the column chamber valve opens. Set the appropriate values of the SEM imaging parameters HV ( $20 \mathrm{kV}$ ), aperture $(120 \mu \mathrm{m})$, high current mode ON, scan speed (e.g., 5), magnification (e.g., 3,000) and noise reduction (pixel average).

3. Focus the electron beam on the sample surface at WD in the range between 12 and $22 \mathrm{~mm}$. 
4. Switch off the electron beam acceleration voltage and close the column chamber valve.

5. Turn on the power supply for the EBSD detector. Move the EBSD detector from parking position to measuring position in the SEM chamber.

6. Open the column chamber valve and switch on the electron beam acceleration voltage. Re-focus the electron beam on a region of interest on the sample surface.

7. Open the EBSD control software and load the calibration file for the chosen WD.

8. Set up the measurement in the EBSD control software according to the operating manual.

9. Perform the background acquisition according to the EBSD operating manual.

10. Read out the position of the EBSD pattern center and the detector distance for the chosen WD from the EBSD control software.

11. Focus the electron beam in a region of the sample adjacent to the region of interest for the actual measurements. Leave the beam to scan there for about $1 \mathrm{hr}$ for stabilization.

12. Check the amount of beam drift which must not exceed more than $1 \mu \mathrm{m}$ during the whole EBSD measurement.

13. Navigate the electron beam to the region of interest and re-focus.

14. Schedule line scans parallel to the tilt axis in the region of interest. Use the beam mapping (not stage mapping) setting. Select appropriate values for the number of steps (e.g., 100), step size (e.g., $50 \mathrm{~nm}$ ), exposure time (e.g., $43 \mathrm{msec}$ ), frame average (e.g., 10) and detector binning (e.g., $2 \times 2)$.

15. Be sure to select "save all images". Disable indexing to speed up the measurement.

16. Run the line scans until the last scan is finished.

17. Switch off the electron beam acceleration voltage and close the column chamber valve.

18. Retract the EBSD detector from the measuring position to park position and turn off the EBSD control unit.

19. Vent the chamber, remove the sample and evacuate.

\section{Analysis of the CL-data:}

1. Check the grating calibration by comparing the spectral positions of known spectral lines (e.g., peaks of luminescence lines of the bulk and unstrained GaAs test sample with the data in the literature) and modify the wavelength calibration if necessary.

2. Correct the recorded CL spectra (intensity vs. wavelength) with respect to the wavelength-dependent sensitivity of the optical system which consists of blaze grating and PMT by dividing the measured intensity by the corresponding sensitivity value at each measuring point.

3. Extract the grey value of each pixel of a CL image and average the grey values for each region of interest in the image, at least on the scale of the electron interaction volume in the sample.

4. Determine the $C L$ intensity for the region of interest from a calibration curve of the averaged grey values of the $C L$ images, depending on the $\mathrm{CL}$ intensity for given contrast and brightness values of the PMT.

5. Evaluate the mean $C L$ intensity $I_{C L}$ of the whole $C L$ image and the dark signal $I_{0}$ of the $C L$ detector.

6. Calculate the contrast $C_{C L}$ between bright and dark appearing regions in the $C L$ image by the following equation:

$C_{C L}=\frac{I_{C L, \text { bright }}-I_{C L \text { dark }}}{I_{C L}-I_{0}}$

\section{Analysis of the EBIC-data:}

1. Extract the grey value of each pixel of an EBIC image and average the grey values for each region of interest to quantify spatial variations of the EBIC intensity in terms of an EBIC contrast.

2. Determine the EBIC contrast profile from the spatially resolved grey values due to C. Donolato and R.O. Bell ${ }^{18}$.

3. Calculate the area and variance of the EBIC contrast profiles.

4. Determine physical parameters such as the diffusion length and the surface recombination velocity of minority charge carriers from the calculated area and variance of the EBIC contrast profile according to the rules given by Donolato ${ }^{14}$.

\section{Analysis of ccEBSD Experiments}

1. Use the in-house written software "ccEBSD" programmed by Paul Chekhonin for the evaluation of the recorded EBSD patterns.

2. Choose an appropriate EBSD pattern that will act as the reference pattern from a measuring point that should be located in a specimen area absent of strains.

3. Apply at least 15 widely distributed regions of interest on the pattern.

4. Use the coordinates of the pattern center and the detector distance which were determined by the EBSD control software for the further evaluation.

5. Enter the rest of the parameters relevant for the evaluation, i.e., elastic constants of the analyzed material, number of steps in the line scan and step size, detector size and its resolution in pixels, detector and specimen tilt angles as well as the detector binning.

6. Use a band pass filter during the calculation of the 2-dimensional Fourier transformation (inner filter of 6 pixels and outer filter of 40 pixels).

7. Run the evaluation program.

8. Read out the numerical results from the evaluation text file "protocol.txt" using a conventional spreadsheet program when the calculation is finished. 


\section{Representative Results}

The structural, electrical and optical properties of extended defects in a semiconductor material were studied by different experimental methods in the scanning electron microscope. Generally, it is possible to investigate these properties on the same sample, and with some efforts concerning the sample preparation, even on a distinct single defect like a grain boundary or a localized arrangement of dislocations. However, it should be noted that due to the specific interaction products of the primary electron beam with the semiconductor material used for the inspection of physical defect properties, the spatial resolution which can be achieved by CL, EBIC or ccEBSD investigations differs from each other. In Figure 1, schematic drawings are given for an appropriate set-up of the SEM suited for CL measurements at low temperatures (Figure 1A), the assembly for EBIC investigations (Figure 1B) as well as the layout of the main hardware elements necessary for (cC)EBSD tests (Figure 1C).

All the representative results given here are obtained for silicon as a showcase for a semiconductor material with indirect electronic band structure. This band structure impedes all luminescence measurements due to the low probability of radiative transitions in comparison to semiconductors with direct band gap structures. To realize sufficient luminescence intensity for statistically assured results is challenging. In the following, the experimental procedures are described for the investigation of dislocations induced by plastic deformation as well as by liquid phase re-crystallization in silicon single crystals. Additionally, investigations on a silicon bi-crystal with twin grain boundaries and a low-angle grain boundary are presented.

Figure 2A shows an example of the appropriate positioning of a sample on the indium foil to guarantee a good thermal contact to the cryosample holder in which the temperature is measured by the thermocouple. It was proven experimentally that for silicon, a sample thickness of about 200 to $500 \mu \mathrm{m}$ is well suited for cryo-CL investigations at temperatures down to $5 \mathrm{~K}$. The CL spectra given in Figure 2B were measured for a Si single crystal in the virgin state, after plastic deformation and after an additional annealing. The electron beam in the SEM was run at an electron beam acceleration voltage of $20 \mathrm{kV}$ and a probe current of approximately $45 \mathrm{nA}$ in the defocused scanning mode, which results in a high CL intensity due to electron-hole pair generation in a large volume (about $\left.(450 \times 250 \times 3) \mu \mathrm{m}^{3}\right)$ with moderate excitation density. In this scanning mode, the sample surface is actually on $W D=15 \mathrm{~mm}$ but electronically a WD $=0$ is adjusted. For $C L$ imaging, of course, the electron beam has to be focused which yields a spot diameter of the electron beam on the sample surface of a few nm but with the same penetration depth of some $\mu \mathrm{m}$ for the primary electrons as in the defocused scanning mode. The acquisition time per image with a store resolution of 1,024 $x 768$ pixels was about $10 \mathrm{~min}$ in pixel averaging mode at scan speed 14 of the electron beam. It was calculated and experimentally confirmed that for the defocused scanning mode the temperature of the sample region under investigation is increased no more than some $0.1 \mathrm{~K}$ by heat energy transfer due to the electron beam. In the focused mode, the local sample heating strongly depends on the thermal conductivity which in

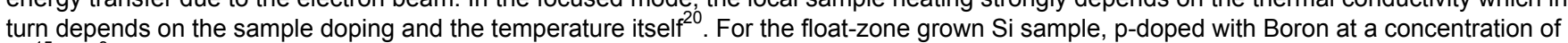
$10^{15} \mathrm{~cm}^{-3}$, in the focused scanning mode, a local temperature increase $\Delta \mathrm{T}$ of about $2 \mathrm{~K}$ occurred at a cryostat temperature of $5 \mathrm{~K}$, and of $\Delta \mathrm{T} \approx 0.3$ $\mathrm{K}$ at $25 \mathrm{~K}$.

To study the optical properties of dislocations, a bulk Si sample was subjected to a plastic deformation at a pressure of $16 \mathrm{MPa}$ at $800{ }^{\circ} \mathrm{C}$ followed by a second deformation step at $295 \mathrm{MPa}$ at $420^{\circ} \mathrm{C}$. The slip lines, shown in Figure $\mathbf{2 C}$ on the surface of a part of the deformed sample, are caused by dislocation glide processes on two different 111-oriented slip planes. The slip lines can be visualized by backscattered electrons (BSE). The slip lines indicate the traces of the lattices planes on which most of the dislocations are aligned. Monochromatic CL (mono$\mathrm{CL}$ ) images (Figures 2D and 2E) were acquired at energetic positions of the D4 and D3 luminescence bands and do not suffer significantly from the surface topography profile caused by slip lines. This was verified by CL investigations after a careful surface polishing which showed the same nearly unaltered luminescence stripe pattern as on the originally wavy sample surface, where the CL intensity stripe patterns are parallel to the slip plane traces. If it is planned to analyze the local distribution of the CL luminescence intensity quantitatively from the image, then the $\mathrm{CL}$ image has to be recorded in the linear range of the relation between $\mathrm{CL}$ signal and grey value. This relation can be determined experimentally by measuring the correlation between image grey value and absolute signal of the photomultiplier at given contrast and brightness values for the detector. On the contrary, if it is intended to visualize small variations of CL intensity on the sample surface, then for best results, a non-linear signal-to-grey value relation should be applied already during the imaging process in the SEM. The spatial resolution of a CL image on a bulk Si sample at low temperatures is determined by the size of the interaction volume of the primary electrons in the sample, because the size of this interaction volume is only marginally smaller than the volume for the radiative recombination of the electron-hole pairs ${ }^{21}$. The diameter of the interaction volume for a focused and stationary beam is about $3 \mu \mathrm{m}$ under the given experimental conditions ${ }^{22}$.

The estimation of the strain field surrounding extended defects by ccEBSD requires the recording of Kikuchi patterns with sufficient quality even on highly strained sample regions. An example is given in Figure 3A. To get these patterns, the sample surface should be free of undesirable surface layers (oxides, carbon contamination, etc.). Good results can be achieved with the following experimental parameters: electron beam at $20 \mathrm{keV}$ and $12 \mathrm{nA}$, tilt of sample surface normal between $60^{\circ}$ and $70^{\circ}$ to the incident beam at WD $=15 \mathrm{~mm}, 2 \times 2 \mathrm{EBSD}$ detector pixel binning which yields a resolution of $672 \times 512$ pixels, amplification gain of the signal set to high, exposure time between 20 and 43 msec per frame on the EBSD detector, averaging over five to ten frames per measuring point and storage of the Kikuchi pattern as images for each measuring point without indexing. The total acquisition time for one Kikuchi pattern can be estimated from the exposure time multiplied by the number of frames plus a few $10 \mathrm{msec}$ due to the time necessary for beam shift, read out and storage. A value of $50 \mathrm{~nm}$ turned out experimentally to be a good minimum step size between two sample positions within the EBSD mapping. This is in agreement with recent theoretical considerations ${ }^{23}$ concerning the achievable resolution for electron diffraction contrast. To avoid beam drift during the EBSD mapping, it is recommended to wait at least $15 \mathrm{~min}$ with the beam scanning in the immediate neighborhood of the region of interest before running the map. It was found that only EBSD line scans parallel to the sample tilt axis provide realistic strain data with a reference pattern on the same line. Otherwise, a very careful determination of the actual sample tilt angle is needed, or alternatively the length of a line scan perpendicular to the tilt axis must be limited to a few $\mu \mathrm{m}$. 
The Kikuchi pattern stored as 8-bit JPEGs were evaluated by Fourier transformation (FT) and cross-correlation with a program "ccEBSD" written by one of the authors (PC). The program is based on the algorithm developed by Wilkinson et.al. ${ }^{6}$, described in detail in ref. 19 . In the Kikuchi pattern, several (15 - 19) sub-patterns (128 x 128 pixels) have to be defined with characteristic features as bright band crossings (cf. Figures 3A and 3B). All sub-patterns have to be analyzed by FT. A band pass filter has to be applied to all of the FT images (inner radius 6 pixels for low frequencies, outer radius 40 pixels for higher frequencies) to set all values to zero outside the band pass filter in the Fourier space (cf. Figure 3C). Then the cross-correlation (cc) function (Figure 3D) has to be calculated between the FT of each sub-pattern with the respective FT of the sub-pattern (Figures 3E and 3F) from the reference Kikuchi pattern. From the positions of the peaks in the cc-functions (Figure 3D), the relative displacements of the sub-patterns can be determined. Using these displacements, the normal and shear strain components can be calculated. If the material dependent elastic constants are known, also the stress components can be determined. In the notation by Voigt, these constants are $\mathrm{C}_{11}=165.7 \mathrm{GPa}, \mathrm{C}_{12}=63.9 \mathrm{GPa}$ and $\mathrm{C}_{44}=79.9 \mathrm{GPa}$ for $\mathrm{Si}$ with a cubic lattice ${ }^{24}$. The combination of the results from all sub-patterns of one Kikuchi pattern improves the accuracy of the strain evaluation. The statistical error determined from a ccEBSD line scan on a defect free region in a silicon single crystal is found to be $2 \times 10^{-4}$ for all the strain tensor components. Nevertheless, to obtain quantitative results for the case of extended defects, the choice of a Kikuchi pattern as reference pattern is important. If, for example, the sample is completely covered by dislocations as shown in Figure 2, sophisticated procedures that are proposed by Jiang et al. ${ }^{25}$ could be applied to find out the appropriate reference pattern.

The situation for the use of ccEBSD is easier for a Si wafer ([001]-surface orientation) treated by a high-energy electron beam to induce a liquid phase re-crystallization (see Figure 4). Around the track of re-crystallization, slip lines are visible in the BSE image indicating a dislocation movement on slip planes with traces parallel to the edges of the image (Figure 4A). The CL investigations were performed under the same experimental conditions as for the plastically deformed sample. The mono-CL images, recorded at the energies of the band-to-band transition and of the D4 and D2 dislocation luminescence bands (Figures 4B, 4C and 4D, respectively), show the spatial distribution of the extended defects caused by the re-crystallization procedure. A local anti-correlation between the band-to-band transition and the $D$ line luminescence bands can be inferred from the mono-CL images. This is supported by the CL spectra (Figure 4E) which were measured at sample positions 1,2 and 3 (cf. Figure 4A) in spot mode of the electron beam. From the ccEBSD investigations performed as a line scan in front of the recrystallization track (white line in Figure 4A), the local strain tensor components along the line scan could be determined (Figures $\mathbf{4 F}$ and $4 \mathbf{G}$ ). It was proven, that within the statistical error, the values do not depend on which particular Kikuchi pattern was used as the reference pattern if this pattern is situated in a region where the band-to-band transition is dominant. The dislocation related electronic transitions appear when the sum of normal strains $\operatorname{Tr}(\varepsilon)$ exceeds a value of $5 \times 10^{-4}$. Because $\operatorname{Tr}(\varepsilon)$ is not equal to zero for the scan in a region of about $150 \mu \mathrm{m}$ length close to the re-crystallization track, there is a mean lattice dilation in a volume near to the sample surface. According to the linear theory of elasticity, the normal stress $\sigma_{33}$ is equal to zero as presupposed in the evaluation program "ccEBSD". If there is a crack on the EBSD line scan, then a ccEBSD evaluation cannot be performed over the whole scan with one reference pattern due to abrupt variations of the Kikuchi pattern caused by the geometrical effects of the crack.

What can be achieved in principle by the experimental methods described for the investigation of structural, optical and electrical properties of grain boundaries in $\mathrm{Si}$ is shown in Figure 5 for a Si bi-crystal of p-type doping with a Boron concentration of $10^{17} \mathrm{~cm}^{-3}$. The conventional EBSD map yields the full information on the crystal orientation at each point of the map where only the indexing of the Kikuchi pattern is performed immediately after the pattern acquisition by the acquisition software. Additionally, also the type of grain boundaries can be displayed by the conventional EBSD data managing program (Figure 5A). For the detection of a LAGB, a critical angle has to be defined for the misorientation of the crystal lattice at two adjacent measuring points. A minimum value of $1^{\circ}$ was proven to be appropriate. For the LAGB indicated in the EBSD map, the misorientation angle is $4.5^{\circ}$. The EBIC-image of the same sample area (Figure $5 B$ ) was measured at RT. The incoherent $\Sigma 3$ grain boundaries and the LAGB appear here as dark lines. This effect is caused by the locally increased carrier recombination. From the contrast profile of the EBIC signal across the LAGB (cf., Figure $\mathbf{5 H}$ ), a diffusion length of $(60 \pm 12) \mu \mathrm{m}$ and a recombination velocity of $(4.1$ $\pm 0.4) \times 10^{4} \mathrm{~cm} \mathrm{sec}^{-1}$ were determined for the minority charge carriers in the framework of the model by Donolato ${ }^{14}$. The single dark points in the EBIC image, spread over the entire sample surface and concentrated especially in the vicinity of the LAGB, indicate the positions of threading dislocations. In CL imaging investigations at $4 \mathrm{~K}$, the LAGB appears dark in the mono-CL image at band-to-band transition energies (Figure 5C), as expected, but surprisingly also in a mono-CL image at the energy of the D4 band (Figure 4D) which is usually assigned to dislocations. However, the LAGB looks bright in a mono-CL image at a wavelength of $1,530 \mathrm{~nm}$ corresponding to the D1/D2 luminescence bands (Figure 5E). This luminescence behavior is believed to be induced by point defects in the neighborhood of the dislocations constituting the LAGB. Additionally, the ccEBSD procedure was performed as a line scan across the LAGB to determine its local strain field. The electron beam acceleration voltage was reduced to $10 \mathrm{kV}$ to increase the spatial resolution for the strain determination at the expense of an increased total acquisition time for each Kikuchi pattern. The normal and the shear strain components, shown in Figures $\mathbf{5 F}$ and $\mathbf{5 G}$, respectively, cannot be calculated for the center region of the LAGB (over about $50 \mathrm{~nm}$ ) because double patterns appear that prevent an analysis of the Kikuchi patterns. Moreover, the EBSD patterns on both sides of the LAGB have to be correlated with two different reference patterns because the crosscorrelation method can only be applied for small variations of the diffraction pattern. So, two reference patterns were collected on the left hand side and on the right hand side of the LAGB due to the large misorientation angle between the two sub-grains. Nonetheless, it is exciting that the strain components behave symmetrically on both sides of the LAGB. The diagrams for the position dependence of the strain components show that the range of the strain field of the LAGB extends to about $350 \mathrm{~nm}$ into both sub-grains. On the contrary, the diagram of the locally varying contrast in the band-to-band transition mono-CL image, and of the EBIC signal contrast in the EBIC image (Figure 5H), indicates that the influence of the LAGB on the luminescence signal and on the EBIC signal ranges up to $\pm 10 \mu \mathrm{m}$ and $\pm 1.5 \mu \mathrm{m}$ from the center of the LAGB, respectively. This verifies the statement from the beginning that the local resolution for the investigation of different properties of extended defects strongly depends on the experimental method and parameters applied. 


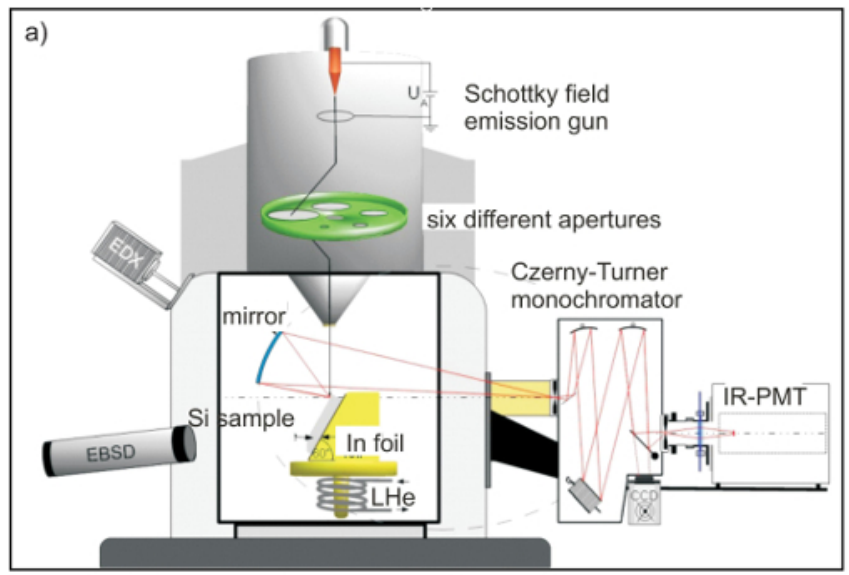

SEM with

CL equipment

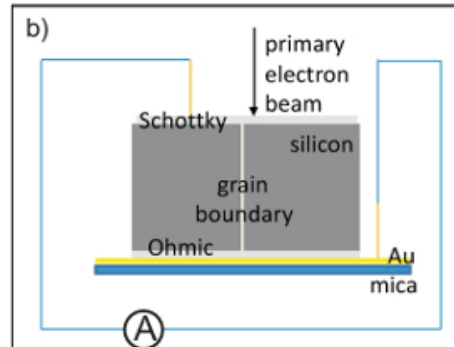

\section{EBIC}

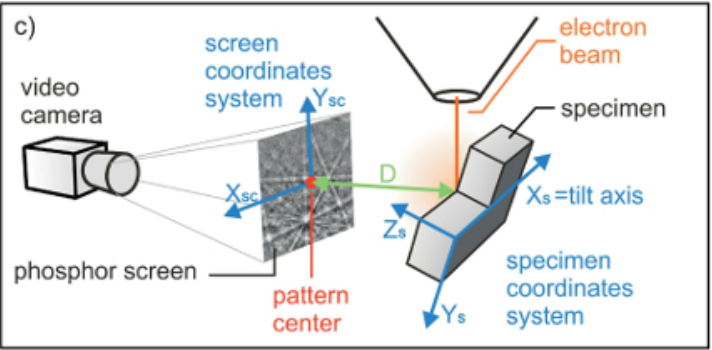

Figure 1. Set Up for CL, EBIC and ccEBSD Measurements. (A) SEM with field-emission gun, different apertures for imaging and analysis, the sample on the cryo-sample holder, the CL light-collecting mirror, the monochromator and the IR-PMT for the infrared light, (B) Schottky contact of the sample for EBIC investigations and (C) set-up for the formation and storage of a Kikuchi pattern which can be analyzed numerically to get information on the crystal orientation as well as on crystal lattice distortions by ccEBSD. Please click here to view a larger version of this figure. 

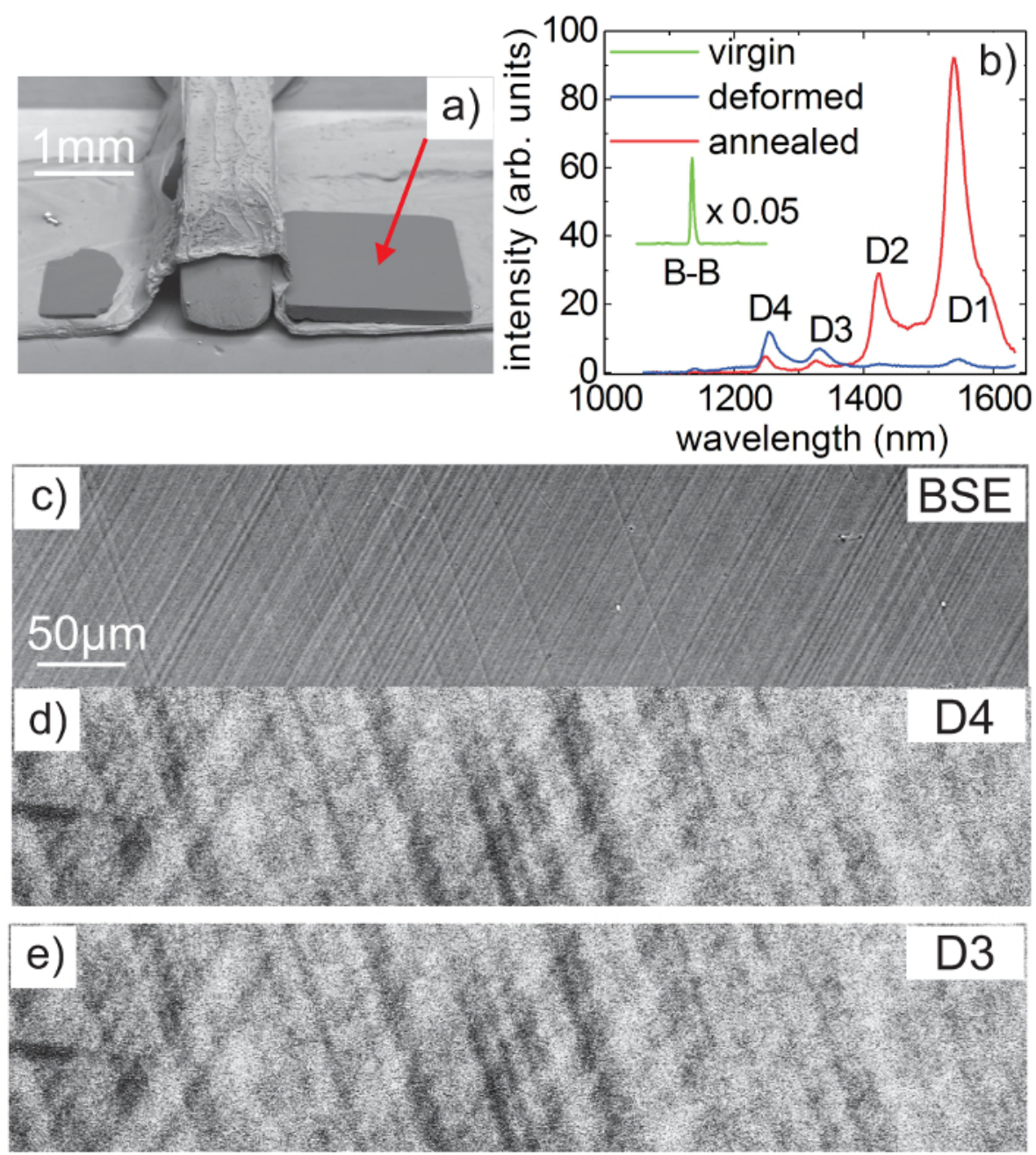

Figure 2. CL Spectral and Imaging Investigations on a Plastically Deformed Silicon Single Crystal. (A) Silicon samples on indium foil positioned on the cryo-sample holder. (B) The CL-spectra measured for a high purity Si single crystal (virgin), for a plastically deformed sample, and after additional annealing. The characteristic transitions in the spectra are labeled as usual with B-B for a band-to-band transition, and D1 to D4 for dislocation induced luminescence bands. (C) Slip lines on the surface of the deformed Si crystal (marked by red arrow in Figure 2A) imaged by back-scattered electrons (BSE). These results show plastic deformation for different slip systems. In Figures 2D and 2E, the mono$\mathrm{CL}$ images for the D4 line and D3 line are shown, respectively, with each measured for the same sample region below that shown in the BSEimage (Figure 2C). Please click here to view a larger version of this figure. 
Kikuchi pattern on sample position

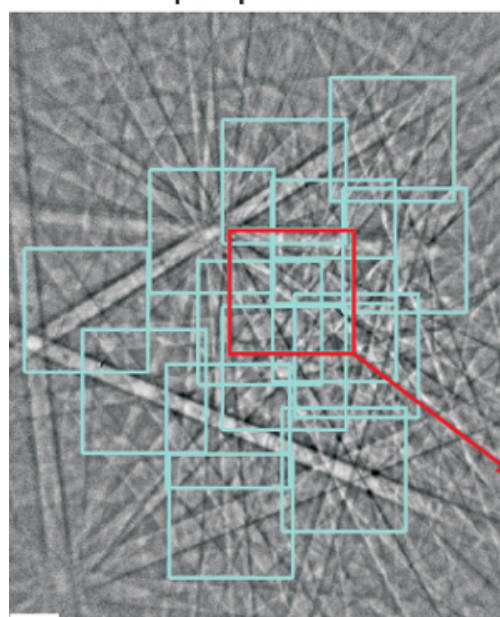

a)

\section{sub-pattern filtered Fourier- \\ transformation}

\section{from reference pattern}
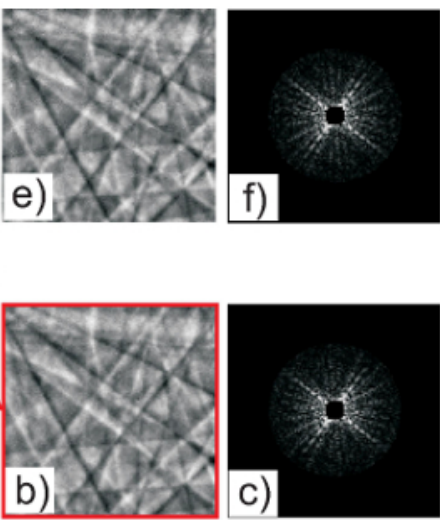

d)

cross-

correlation

function

Figure 3. Images Visualizing Steps in the Course of ccEBSD Analysis. (A) Full Kikuchi pattern from actual sample position with sub-pattern. (B) One of the sub-patterns and (C) its filtered Fourier transformation. (E) The corresponding sub-pattern from a reference position on the sample and (F) its filtered Fourier transformation. (D) The cross-correlation function (CCF) calculated from the Fourier-transformations of the sub-pattern. The brightness of the CCF was increased by $20 \%$ to visualize the details. Please click here to view a larger version of this figure. 

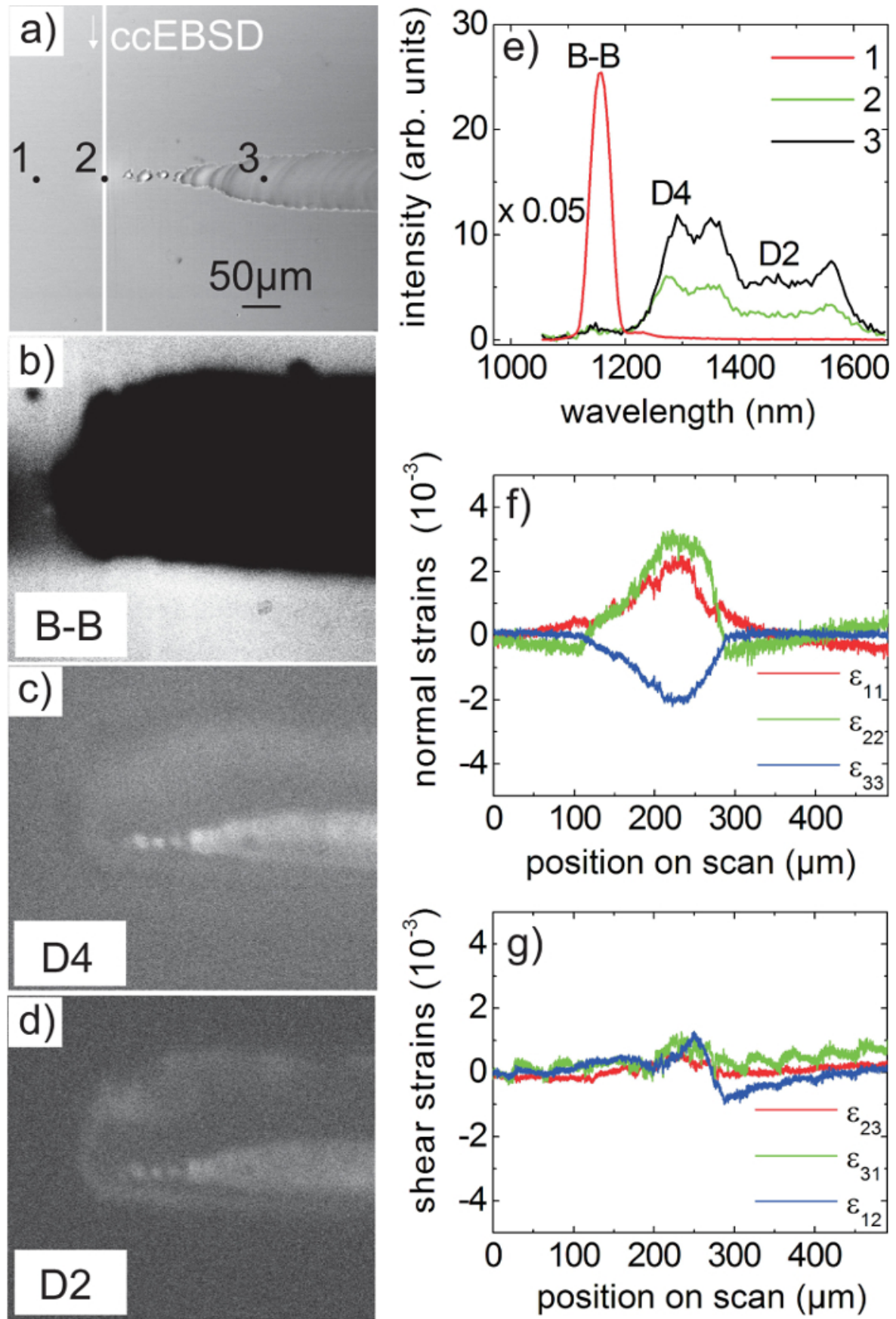

Figure 4. CL and ccEBSD Investigations for a Si Wafer After Re-crystallization. (A) BSE image from the surface of a Si wafer with a track of re-crystallized material after treatment by a high-energy electron beam. Positions of spots 1,2 and 3 for CL spectral investigations are marked as well as the line with direction arrow where the ccEBSD scan was performed. (B-D) Mono-CL images of the sample region shown in (A), taken at the energetic positions of the band-to-band transition (B), D4 (C) and D2 (D) luminescence band. (E) CL spectra measured at the spots 1, 2 and 3. The normal $(\mathbf{F})$ and the shear strain components $(\mathbf{G})$ along the line scan in $(\mathbf{A})$, calculated from ccEBSD investigations. Please click here to view a larger version of this figure. 


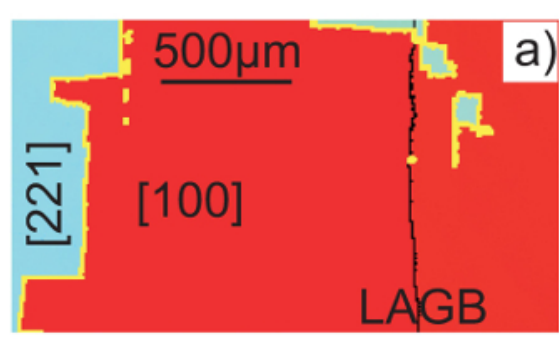

\section{EBSD}
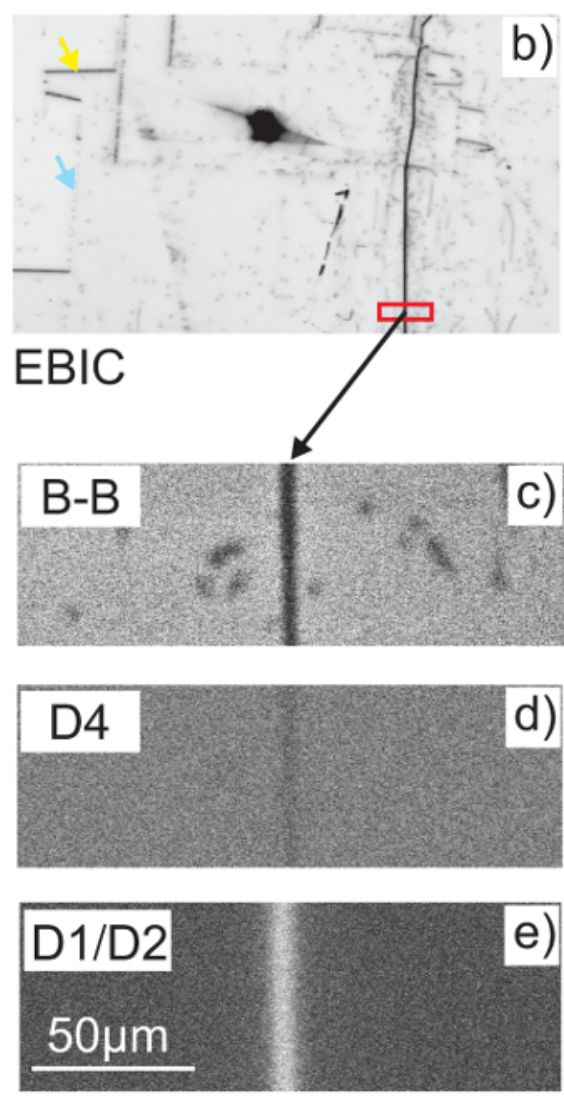

$\mathrm{CL}$
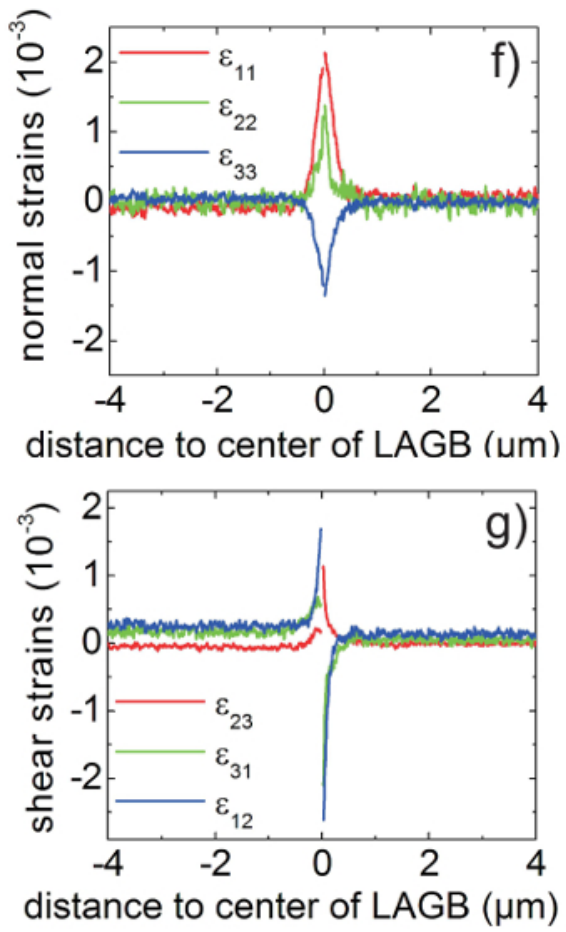

\section{ccEBSD}

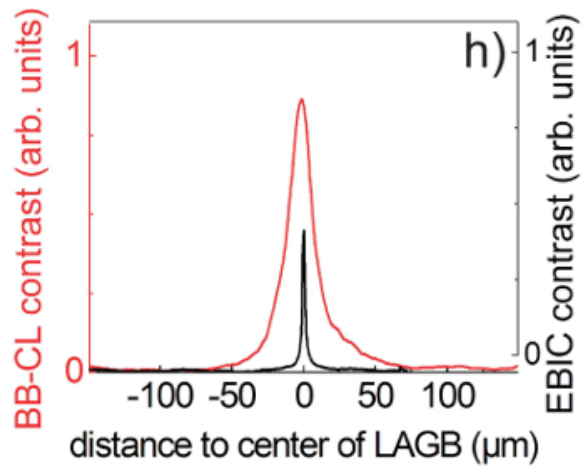

$\mathrm{CL}$ and EBIC contrast

5. EBSD, EBIC, CL and CcEBSD Investigations on a Silicon Bi-crystal with HAGBs and LAGB. (A) EBSD orientation map on a Si bicrystal with twin grain boundaries in yellow and a LAGB in black. The orientation of the normal of the grain surface is indicated. (B) EBIC image at RT of the sample area in (A) where coherent (yellow arrow) and incoherent (blue arrow) twin grain boundaries are indicated. (C-E) The mono$C L$ images at energies of $B-B(C)$, D4 (D) and D1/D2 (E) belong to the LAGB region which is marked by a red rectangle in the EBIC image (B). The normal $(\mathbf{F})$ and the shear strain components $(\mathbf{G})$ calculated from ccEBSD investigations across the LAGB. $(\mathbf{H})$ Comparison of the contrast found in the B-B mono-CL image at $4 \mathrm{~K}$ and in the EBIC image at RT across the LAGB. Please note the different scaling on the $x-c o o r d i n a t e$ in the strain component diagrams and in the CL- and EBIC-contrast diagram. Please click here to view a larger version of this figure.

\section{Discussion}

The SEM offers the possibility to locate extended defects in the semiconductor material as well as to characterize their structural, optical and electrical properties by the application of ccEBSD, CL and EBIC investigations. In general, it is not possible to perform all three methods simultaneously on the same sample. However, a combination of results obtained by the different complementary investigation methods, when performed in a reasonable sequence, leads to a deeper understanding of the physical nature of the effects caused by extended defects.

For the CL measurements giving information on the optical properties of extended defects, a critical step in the protocol is the sample positioning procedure (step 1.6) due to undesired annealing of defects in the sample during the heating of the indium foil (which ensures a good thermal and electrical contact of the sample with the sample holder). An alternative to the procedure proposed is to mount the sample onto the sample holder by conductive silver paste at RT. However, from experience it is known that the organic solvent in the paste can cause carbon contamination on the sample surface during the scan in the SEM. The contamination degrades the quality of the CL images as well as of the EBSD diffraction 
patterns. Additionally, the step 4.21 requires special attention, where an abrupt rise of the luminescence intensity of silicon can occur during the cooling-down of the sample. This can harm the performance of the photomultiplier. On the contrary, for the case of unexpected low luminescence intensity for the actual sample, one should try to improve the adjustment of the light-collecting mirror (protocol No. 4.23) because the preliminary mirror alignment was performed on a test sample at RT in a slightly different range of wavelength.

Concerning instrumental limitations of the method, one has to take into account that at very low temperatures the stage with the sample can be moved only by $\pm 5 \mathrm{~mm}$ in the $\mathrm{x}$ - and $\mathrm{y}$-directions which restricts the area of the samples under investigation. This limitation is due to the danger of brittle fracture of the He transfer tube. The sample dimensions for cryo-experiments given in 1.1 and 1.2 are also limited by experimental conditions. So the surface area of the samples should be adjusted to the size of the sample holder to ensure an optimal thermal contact on the heat sink. The low recommended thickness of the silicon samples limits the temperature gradient in the sample for the cryo-experiments. For a sample thickness of $200 \mu \mathrm{m}$, the temperature in the center of the interaction volume for the primary electrons in the surface region was found to be increased by less than $5 \mathrm{~K}$ in comparison to the temperature measured at the surface of the sample holder. The high scan speed and the low magnification proposed only for the cool-down procedure in steps 4.5 and 4.17 , ensure that the region of interest is kept clean. This is because of the heat transfer by the scanning electron beam which maintains a temperature always slightly above the temperature of the rest of the sample regions which act as condensation trap for residual gas in the SEM chamber. Generally, all the parameters listed in step 4.24 for $\mathrm{CL}$ spectroscopy are optimized for the measurement of the so called $D$ line luminescence in bulk silicon by the experimental set up according to the equipment list. The parameters have to be adapted if investigations of the luminescence are to be carried out on other semiconductor materials.

Independent of the energy range of the luminescence observed, a further limitation of the CL measurements results from the light-collecting mirror because light coming from radiative recombination processes in the whole recombination volume is collected by the mirror and thus determines the grey value of the corresponding $\mathrm{CL}$ image pixel which is assigned to the position of the electron beam on the sample surface. Because the diameter of the recombination volume (which is comparable to the excitation volume) is larger than the pixel size even at low magnification, this effect causes a spatial smearing of the luminescence signal, and, therefore, limits the spatial resolution. Nevertheless, the $\mathrm{CL}$ investigation enables an imaging of the local distribution of mono- or panchromatic luminescence with a medium spectral resolution and could be combined with photoluminescence investigations to give a higher spectral resolution. Recently, as an alternative experimental method to $\mathrm{CL}$ measurements, a microscopic and spectroscopic mapping of dislocation related photoluminescence was proposed by the group of Tajima and co-workers ${ }^{26}$. The spatial resolution of the photoluminescence mapping is clearly lower than in CL images, but the photoluminescence investigations additionally allows the polarization of the deep level emission band correlated to dislocations to be determined in LAGBs with twist and tilt structures ${ }^{27,28}$.

In the case of EBIC investigations, which give insight into the electrical properties of extended defects, there are no alternative methods for the imaging of the locally varying charge-collection efficiency in semiconductor materials with a comparably spatial resolution. However, also for EBIC measurements, critical steps are included in the protocol. So in step 5.13, the variation of the EBIC image with decreasing temperature is expected to arise from the temperature dependent properties of the extended defects. However, the quality of the contacts can change at temperatures below RT and hence influence the EBIC image. Temperature affects the Schottky contact, made with an appropriate layer of Al in the case of $p$-type and with $\mathrm{Au}$ in the case of n-type silicon, because of the different coefficients of thermal expansion separating the contact layer from the silicon substrate. Furthermore, the ohmic contact made by a gallium-indium eutectic is not stable at temperatures below 160 K. Normally, the reduction of the contact quality leads to a strongly decreased EBIC signal for large areas. In this case, the contacts have to be renewed. For EBIC investigations at RT, it is also conceivable that the contacts for the EBSD measurements can be made by bonding the sample to an appropriate carrier board. Another instrumental limitation of EBIC measurements is caused by the protruding of the contact tip holders above the sample surface. To prevent a collision between the contact tip holder and the pole piece of the SEM the WD should be at least $15 \mathrm{~mm}$.

In the experimental procedure for ccEBSD investigations which can be used to estimate the long-range strain field of extended defects, the following steps are critical. The most challenging part of the experiment is the sample preparation, especially the last polishing procedure (protocol No. 3.1) which has to be performed carefully to avoid the generation of additional surface defects. If no Kikuchi pattern can be obtained, often the quality of the sample surface is not sufficient. However, from silicon single crystals with slip lines on the surface after plastic deformation, a good diffraction pattern could be obtained which was well suited for the ccEBSD evaluation procedure. The surface roughness of these samples was analyzed by atomic force microscopy yielding a height variation in the range of up to $500 \mathrm{~nm}$. Therefore, extremely high internal strains or amorphous surface layers seem to be responsible for blurred diffraction patterns rather than the imperfect smoothness of the sample surface. A further issue could be a low signal from the coherently scattered electrons in comparison to the background. Then an increase of the probe current at constant acceleration voltage and/or a more accurate determination of the background signal (protocol step No. 6.12) are helpful. To minimize sample movement during a long-lasting ccEBSD measurement it is recommended to fix the sample mechanically (protocol No. 3.2).

Instrumental limitations for the ccEBSD investigations can arise if the tilt of the sample surface relative to the incident electron beam is realized by the tilt of the stage. There are then strong restrictions for the movement of the sample due to a collision risk with the pole piece and the chamber walls. Furthermore, it is strongly recommended to use only line scans that are parallel to the tilt axis (and thus appear horizontally on the SEM screen), because, first, vertical scans have a large sum error for the internal strains due to the error of sample tilt. Second, during EBSD, the lateral resolution is higher (factor of about 3 for $70^{\circ}$ tilt) along the tilt axis than perpendicular to it. The lower limit for the value of the strain tensor components calculated for Si from ccEBSD investigations is about $2 \times 10^{-4}$ which is the random error. Additionally it must be emphasized that the ccEBSD technique cannot be applied in the presence of large lattice rotations $\left(>4^{\circ}\right)$ referring to the reference point or very close to grain boundaries, where EBSD patterns from different grains overlap. The physical limitation of the cCEBSD investigations concerning the spatial resolution of the strain determination is due to the range of the electron diffraction which was found to be approximately $50 \mathrm{~nm}$ along the sample tilt axis. In comparison with X-ray diffraction experiments for the determination of internal strains, this is a clear advantage because of the significantly larger interaction volume of X-rays even in the case of X-ray $\mu$-diffraction. For semiconductor materials, the investigation of perturbations of the isotropic refractive index by a polarscope can also be applied for the determination of internal stresses, but the spatial resolution of this method is lower than some hundred $\mathrm{nm}^{29}$. An alternative method for the determination of the spatially resolved three-dimensional strain state in crystals is based on the splitting of higher order Laue zones (HOLZ) lines. This method has to be performed in a transmission electron microscope (TEM) using an electron biprism for electron interferometry ${ }^{30}$. However, in contrast to the ccEBSD 
investigations in the SEM, the TEM investigation requires the preparation of a foil from the sample that changes the internal strains due to relaxation effects.

In future studies, ccEBSD measurements will also be performed at low temperatures. This will allow the investigation the structural, optical and electrical properties, not only on the same extended defect, but also at the same temperature.

\section{Disclosures}

The authors have nothing to disclose.

\section{Acknowledgements}

Support of this work by the German Research Foundation (DFG) within the framework of the Research Training Group 1621 is gratefully acknowledged by Paul Chekhonin. All authors are grateful to Dietmar Temmler (Fraunhofer FEP Dresden) for providing the electron beam processed Si samples showing liquid phase re-crystallisation. Special thanks go to Stefan Saager and Jakob Holfeld for the preparation of the figures for the SEM equipment and the EBSD set-up. We thank Michael Stavola for detailed discussions and help with this work.

\section{References}

1. Alexander, H. Dislocations. In: Materials Science and Technology. Schröter, E., ed., Vol. 4 Electronic Structure and Properties of Semiconductors, Chapter 6, VCH Basel, 249-319 (1991).

2. Mahajan, S. Defects in semiconductors and their effects on devices. Acta Mater. 48, 137-149 (2000).

3. Holt, D.B., Yacobi, B.G. Extended Defects in Semiconductors- Electronic Properties, Device Effects and Structures. Cambridge University Press (2007).

4. Hornstra, J. Models of grain boundaries in the diamond lattice. Physica. 25 (1-6), 409-422 (1959).

5. Randle, V. Crystallographic characterization of planes in the scanning electron microscope. Mater. Charact. 34 (1), $29-34$ (1995).

6. Wilkinson, A.J., Meaden, G., Dingley, D.J. High-resolution elastic strain measurement from electron backscatter diffraction patterns: New levels of sensitivity. Ultramicroscopy. 106 (4-5), 307 - 313 (2006).

7. Wilkinson, A.J., Meaden, G., Dingley, D.J. High resolution mapping of strains and rotations using electron backscatter diffraction. Mater. Sci. Tech. Ser. 22 (11), 1271-1278 (2006).

8. Drozdov, N.A., Patrin, A.A., Tkachev, V. D. Recombination radiation on dislocations in silicon. JETP Lett. 23 (11), $597-599$ (1976)

9. Dean, P.J., Williams, G.M., Blackmore, G. Novel type of optical transition observed in MBE grown CdTe. J Phys. D: Appl. Phys. 17 (11), 2291-2300 (1984).

10. Nacke, M., Allardt, M., Chekhonin, P., Hieckmann, E., Skrotzki, W., Weber, J. Investigations on residual strains and the cathodoluminescence and electron beam induced current signal of grain boundaries in silicon. J. Appl. Phys. 115 (16), 163511-1 -163511-8 (2014).

11. Ikeda, K., Sekiguchi, T., Ito, S., Suezawa, M. EBIC and Cathodoluminescence Study of the Bonded Silicon Wafers. Solid State Phenom. 63-64, 481-488 (1998).

12. Sekiguchi, T., Ito, S., Kanai, A. Cathodoluminescence study on the tilt and twist boundaries in bonded silicon wafers. Mater. Sci. Eng. B. 91-92 (4), 244-247 (2002).

13. Lee, W., Chen, J., Chen, B., Chang, J., Sekiguchi, T. Cathodoluminescence study of dislocation-related luminescence from small-angle grain boundaries in multicrystalline silicon. Appl. Phys. Lett. 94, 112103 (2009).

14. Donolato, C. Theory of beam induced current characterization of grain boundaries in polycrystalline solar cells. J. Appl. Phys. $\mathbf{5 4}$ (3), 1314-1322 (1983).

15. Kittler, M. et al. Regular Dislocation Networks in Silicon as a Tool for Nanostructure Devices used in Optics, Biology, and Electronics. Small 3 (6), 964-973 (2007)

16. Kveder, V., Kittler, M., Schröter, W. Recombination activity of contaminated dislocations in silicon: A model describing electron-beam-induced current contrast behavior. Phys. Rev. B. 63, 115208 (2001).

17. Higgs, V., Lighthowlers, E.C., Norman, C.E., Kightley, P. Characterisation of dislocations in the presence of transition metal contamination. Mater. Sci. Forum. 83-87, 1309-13014 (1992).

18. Donolato, C., Bell, R.O. Characterization of grain boundaries in polycrystalline solar cells using a computerized electron beam induced current system. Rev. Sci. Instrum. 54 (8), 1005-1008 (1983).

19. Wilkinson, A. J., Meaden, G. Dingley, D. J. Mapping Strains Using Electron Backscatter Diffraction. In: Electron Backscatter Diffraction in Material Science. Schwartz, A.J., Kumar, M., Adams, B.L. and Field, D.P., eds., chap. 17, 2nd Edition, Kluwer Academics/Plenum Publishers, New York, 231-249 (2009).

20. Childs, G.B., Ericks, L.Y., Powell, R.W. Thermal Conductivity of Solids at Room Temperature and Below: A Review and Compilation of the Literature. National Bureau of Standards. http://digital.library.unt.edu/ark:/67531/metadc13173/m1/1/, (1973).

21. Yacobi, B.G., Holt, D.B. Cathodoluminescence Microscopy of Inorganic Solids. Plenum Press., New York (1990).

22. Everhart, T.E., Hoff, P.H. Determination of Kilovolt Electron Energy Dissipation vs Penetration Distance in Solid Materials. J. Appl. Phys. 42 (13), 5837-5846 (1971).

23. Zaefferer, S., Elhami, N.-N. Theory and application of electron channelling contrast imaging under controlled diffraction conditions. Acta Mater. 75, 20-50 (2014).

24. Schulze, G. Metallphysik. Akademie Verlag., Berlin, (1967).

25. Jiang, J., Britton, T.B., Wilkinson, A.J. Evolution of intragranular stresses and dislocation densities during cyclic deformation of polycrystalline copper. Acta Mater. 94, 193-204 (2015).

26. Inoue, M., Sugimoto, H., Tajima, M., Oshita, Y., Ogura, A. Microscopic and spectroscopic mapping of dislocation-related photoluminescence in multicrystalline silicon wafers. J. Mater. Sci.: Mater. Electron. 19, 132-134 (2008). 
27. Kato, G., Tajima, M., Toyota, H., Ogura, A. Polarized photoluminescence imaging analysis around small-angle grain boundaries in multicrystalline silicon wafers for solar cells. Jpn. J. Appl. Phys. 53, 080303 (2014).

28. Tajima, M. Spectroscopy and Topography of Deep-Level Luminescence in Photovoltaic Silicon. IEEE J. Photov. 4 (6), 1452-1458 (2014).

29. Yablon, A.D. Optical and mechanical effects of frozen-in stresses and strains in optical fibres. IEEE J. Sel. Top. Quant. 10 (2), $300-311$ (2004).

30. Herring, R., Norouzpour, M., Saitoh, K., Tanaka, N., Tanji, T. Determination of three-dimensional strain state in crystals using self-interfered split HOLZ lines. Ultramicroscopy. 156, 37-40 (2015). 